\title{
Atividade física para prevenção e tratamento das doenças crônicas não transmissíveis e da incapacidade funcional
}

\author{
Physical activity to prevent and treat \\ non-communicable chronic diseases \\ and functional disability
}

Christianne de Faria COELHO ${ }^{1}$

Roberto Carlos BURINI²

\section{RESU MO}

A atividade física pode ser efetivar tanto na atenção primária quanto secundária e terciária da saúde. Os objetivos do artigo são analisar a associação entre atividade física e prevenção ou tratamento das doenças crônicas não-transmissíveis e incapacidade funcional e rever os principais mecanismos biológicos responsáveis por essa associação e as recomendações atuais para a prática de exercícios nessas situações. Diversos estudos epidemiológicos mostram associação entre aumento dos níveis de atividade física e redução da mortalidade geral e por doenças cardiovasculares em indivíduos adultos e idosos. Embora ainda não estejam totalmente compreendidos, os mecanismos que ligam a atividade física à prevenção e ao tratamento de doenças e incapacidade funcional envolvem principalmente a redução da adiposidade corporal, a queda da pressão arterial, a melhora do perfil lipídico e da sensibilidade à insulina, o aumento do gasto energético, da massa e força muscular, da capacidade cardiorrespiratória, da flexibilidade e do equilíbrio. No entanto, a quantidade e qualidade dos exercícios necessários para a prevenção de agravos à saúde podem ser diferentes daquelas para melhorar o condicionamento físico. De forma geral, os consensos para a prática de exercícios preventivos ou terapêuticos contemplam atividades aeróbias e resistidas, preferencialmente somadas às atividades físicas do cotidiano. Particularmente para os idosos ou adultos, com co-morbidades ou limitações que afetem a capacidade de realizar atividades físicas, os consensos preconizam, além dessas atividades, a inclusão de exercícios para o desenvolvimento da flexibilidade e do equilíbrio.

Termos de indexação: Atividade física. Doenças cardiovasculares. Doenças crônicas. Exercício físico.

\footnotetext{
1 Universidade Federal de Mato Grosso, Faculdade de Educação Física. Av. Fernando Corrêa da Costa, 2367, Boa Esperança, 78060-900, Cuiabá, MT, Brasil. Correspondência para/Correspondence to: C.F. COELHO. E-mail: <christianne.coelho@hotmail.com>.

${ }^{2}$ Universidade Estadual Paulista Júlio de Mesquita Filho, Faculdade de Medicina de Botucatu, Departamento de Saúde Pública e Centro de Metabolismo em Exercício e Nutrição. Botucatu, SP, Brasil.
} 
938 | C.F. COELHO \& R.C. BURINI

\section{A B S T R A C T}

Physical activity can be effective on primary, secondary and tertiary health care. The aims of this article are to analyze the association between physical activity and prevention or treatment of non-communicable chronic diseases and functional disability and to review the main biological mechanisms responsible for this association and the current recommendations for the practice of physical exercises in these contexts. Many epidemiological studies show a connection between increased levels of physical activity and decreased general death rates and deaths caused by cardiovascular diseases in adult and elderly individuals. Although the mechanisms that relate physical activity to the prevention and treatment of diseases and functional disability are not completely understood, they mainly involve reduction of body fat, decrease in blood pressure, improvement of the lipid profile and insulin sensitivity and an increase in energy expenditure, muscle mass and strength, cardiopulmonary capacity, flexibility and balance. However, the amount and quality of exercises necessary to prevent damages to health may be different from those necessary to improve physical conditioning. Generally speaking, the consensus for the practice of preventive or therapeutic exercises involves aerobic activities and resistance training, preferably in addition to the daily physical activities. Particularly among adult and elderly individuals who have other diseases or disabilities that affect their ability to exercise, the consensual recommendation is to include exercises that improve flexibility and balance, in addition to the abovementioned activities.

Indexing terms: Motor activity. Cardiovascular diseases. Chronic disease. Exercise.

\section{N T R O D U ÇÃ O}

Algumas doenças crônicas não-transmissíveis, como as cardiovasculares, seus fatores de risco metabólicos (diabetes mellitus, hipertensão arterial sistêmica e dislipidemias) e a incapacidade funcional são importantes causas de morbidade e mortalidade entre adultos e idosos ${ }^{1}$.

Em geral, essas doenças são de longa duração, múltiplas, exigem acompanhamento multidisciplinar permanente, intervenções contínuas e requerem que grandes recursos materiais e humanos sejam despendidos, gerando encargos ao sistema público e social. No Brasil, por exemplo, elas respondem por, aproximadamente, 70\% dos gastos assistenciais com a saúde².

Apesar de a herança genética ser fator de grande relevância na determinação da suscetibilidade à doença, o desenvolvimento dessas morbidades se dá, primordialmente, por fatores ambientais e do estilo de vida. Estima-se que 75\% dos casos novos de doenças não-transmissíveis poderiam ser explicados por dieta e inatividade física ${ }^{1}$. O baixo condicionamento cardiorrespiratório, a pouca força muscular e o sedentarismo, por exemplo, aumentam em três a quatro vezes a prevalência da Síndrome Metabólica (SM) 3 .
A atividade física e/ou o exercício físico pode atuar na atenção primária, secundária e terciária da saúde. No entanto, embora a maioria dos mecanismos biológicos associados à redução, tanto da morbimortalidade por agravos não-transmissíveis como da incapacidade funcional, pela prática de exercícios físicos, ainda não estejam completamente entendidos, aqueles já estabelecidos tornam evidentes a associação da atividade física com promoção e recuperação da saúde 4 .

Nesse sentido, algumas recomendações para a prática de exercícios são elaboradas ou reformuladas ao longo dos anos, no intuito de auxiliar os indivíduos ou profissionais a iniciar um programa apropriado de exercícios preventivos ou terapêuticos ${ }^{5-11}$.

O objetivo deste artigo é analisar a associação entre atividade física e prevenção ou tratamento das doenças crônicas não-transmissíveis e incapacidade funcional, rever os principais mecanismos biológicos responsáveis por esta associação e as recomendações atuais para a prática de atividades físicas nessas situações.

As recomendações sobre atividade física na prevenção e no tratamento de doenças crônicas abordadas no artigo e da incapacidade fun- 
cional, foram baseadas nos consensos e nas diretrizes mais atuais sobre o assunto, publicados pelas sociedades nacionais, como a Sociedade Brasileira de Cardiologia, Hipertensão e Diabetes, além dos órgãos internacionais como o United States Department of Health and Human Services, o American College of Sports Medicine, a American Heart Association e a Organização Mundial da Saúde.

Os artigos científicos citados no estudo foram fundamentados em uma extensa procura na base de dados do Highwire Press (2000-2008), Ovid (1990-2008) e SciELO (2000-2007). Além disso, foram selecionados estudos epidemiológicos do tipo caso-controle e coorte, considerados clássicos na área de atividade física e saúde. O Ministério da Saúde foi utilizado como fonte de dados epidemiológicos, por melhor representar a população brasileira.

Para a recuperação de informação nas bases de dados utilizaram-se as seguintes palavras-chave, individualmente ou combinadas: physical activity, health, functional ability, chronic disease, recommendation, mortality.

\section{Atividade física preventiva e terapêutica}

\section{Evolução e recomendações}

Na metade do século passado, as recomendações de atividade física para melhorar o condicionamento físico e ter benefícios à saúde foram baseadas em comparações sistemáticas dos efeitos de diferentes protocolos de exercícios.

Em 1978 foi publicado o posicionamento do Colégio Americano de Medicina Esportiva intitulado "A quantidade e qualidade dos exercícios para o desenvolvimento e manutenção do condicionamento em adultos saudáveis", que estabelecia os exercícios necessários para que os adultos saudáveis mantivessem ou melhorassem a aptidão cardiorrespiratória e a composição corporal. Eram recomendadas atividades aeróbias, como caminhadas, corridas, ciclismo, realizadas na freqüência de 3 a 5 dias/semana, com intensidade de $60 \%$ a $90 \%$ do $\mathrm{VO}_{2}$ máximo e duração de 15 a 60 minutos $^{4}$.

Apesar de essas recomendações terem sido reformuladas em 1990, com a inclusão dos exercícios de força e resistência muscular, com o passar do tempo o interesse em melhorar o estado geral de saúde, e não apenas a aptidão cardiorrespiratória, muscular e/ou a composição corporal das pessoas, despertou maior atenção dos pesquisadores para o fato de que o volume de atividades, e não necessariamente a intensidade dos esforços, seria mais importante na promoção da saúde 4 .

Assim, em 1995, o Centro de Controle e Prevenção de Doenças (CDC) e o Colégio Americano de Medicina Esportiva (ACSM) criaram uma recomendação populacional que preconizava que "todos os indivíduos deveriam realizar atividades físicas de moderada intensidade, contínuas ou acumuladas, em todos ou na maioria dos dias da semana, totalizando, aproximadamente, $150 \mathrm{mi}$ nutos/semana ou 200kcal por sessão" 5 .

Tal recomendação 5 , apoiada pela Organização Mundial de Saúde e pelo Ministério da Saúde do Brasil, é atualmente preconizada para a prevenção de algumas doenças crônicas e foi criada na tentativa de aumentar o reconhecimento tanto profissional quanto público dos benefícios à saúde, associados às atividades físicas moderadas; chamar a atenção para a quantidade e a intensidade mínima de atividade física necessária para atingir esses benefícios e encorajar as pessoas a se torna-rem mais ativas e não necessariamente mais condicionadas, permitindo a inclusão de programas mais flexíveis em suas vidas cotidianas.

No entanto, após a publicação desse documento houve muitos erros de interpretação, ou seja, as pessoas continuaram acreditando que apenas as intensidades vigorosas de exercícios poderiam gerar benefícios ou, ainda, que as atividades físicas leves do cotidiano fossem suficientes para promover saúde ${ }^{6}$.

Por esse motivo, em 2007, essa recomendação foi reformulada na tentativa de esclarecer muitos pontos que ficaram implícitos no docu- 
mento anterior. Foi estabelecida a freqüência mínima de 5 vezes por semana e 30 minutos de duração para intensidades moderadas, 3 vezes por semana e 20 minutos para as vigorosas, podendo estas ser complementares. O programa moderado pode ser acumulado em sessões de 10 minutos, no mínimo. Foram incorporados os exercícios de força muscular por, pelo menos, duas vezes por semana ${ }^{6}$.

Além disso, ficou esclarecido que as atividades leves do cotidiano devem ser somadas ao programa, e que maiores quantidades de atividade física geram maiores benefícios, embora a dose-resposta para muitas condições clínicas ainda não tenha sido estabelecida ${ }^{6}$.

Nota-se, por esses documentos, que existe uma importante distinção entre atividade física para a prevenção de doenças crônicas e para o fitness. Assim, a quantidade e a qualidade dos exercícios, necessária para obter benefícios à saúde, podem ser diferentes das recomendadas para atin-gir o bom condicionamento físico.

Ainda, as recomendações também podem ser diferentes quanto ao tipo, à intensidade, à freqüência e à duração das atividades físicas necessárias para o tratamento de doenças. Enquanto as recomendações populacionais parecem ser adequadas para a prevenção de diabetes mellitus, hipertensão arterial sistêmica e dislipidemias em adultos saudáveis ${ }^{5}$, o tratamento não medicamentoso dessas doenças requer uma prática mais sistemática de atividades físicas (Quadro 1).

No caso da obesidade, por exemplo, a análise da Estratégia Global para Alimentação, Atividade Física e Saúde ${ }^{1}$ sugere que o aumento do nível de atividade física, por si só, seja insuficiente para perda ou manutenção do peso de pessoas obesas. As recomendações americanas ${ }^{7}$ apontam que a prevenção do novo ganho do peso perdido por obesos pode requerer 60 a 90 minutos diários de atividades moderadas, e que a transição de sobrepeso à obesidade pode ser prevenida com 60 minutos diários dessas atividades.
Para os indivíduos portadores da síndrome metabólica, a $1^{\text {a }}$ Diretriz Brasileira de Diagnóstico e Tratamento ${ }^{3}$ propõe a prescrição individualizada de treinamento, que inclui exercícios aeróbios de moderada intensidade, preferencialmente aliados aos exercícios resistidos.

O tratamento não medicamentoso dos pacientes diabéticos ${ }^{8}$, hipertensos ${ }^{9}$ e dislipidêmicos ${ }^{10}$ também deve incluir exercícios físicos aeróbios, de intensidade moderada e freqüência superior a 3 vezes por semana, além dos exercícios de resistência muscular localizada, em alguns casos (Quadro 1).

No caso da incapacidade funcional, a recomendação para sua prevenção é direcionada aos indivíduos com idade superior a 65 anos, ou aos adultos de 50 a 64 anos que apresentam co-morbidades ou limitações que afetem a capacidade de realizar atividades físicas ${ }^{11}$.

Assim como nas recomendações populacionais, são indicadas para adultos saudáveis atividades aeróbias e de força muscular. A principal diferença é que para a prevenção da incapacidade funcional, atividades para o desenvolvimento da flexibilidade e do equilíbrio são encorajadas $^{12}$.

O Quadro 1 sumariza as recomendações atuais de atividades física quanto ao tipo, à intensidade, à freqüência e à duração mais indicadas para a prevenção e/ou tratamento de algumas doenças crônicas não-transmissíveis e incapacidade funcional.

Ressalta-se que as recomendações de atividades físicas não devem ser entendidas como receitas de bolo. Há a necessidade de levar em consideração a individualidade biológica, idade, sexo, estado de saúde, objetivos e preferências dos indivíduos.

\section{Impacto da atividade física na prevenção e no tratamento de doenças cardiovasculares e seus fatores de risco}

A importância terapêutica e preventiva da atividade física (AF) foi evidenciada por meio das 
Quadro 1. Recomendações atuais para atividade física ou exercícios físicos preventivos e terapêuticos.

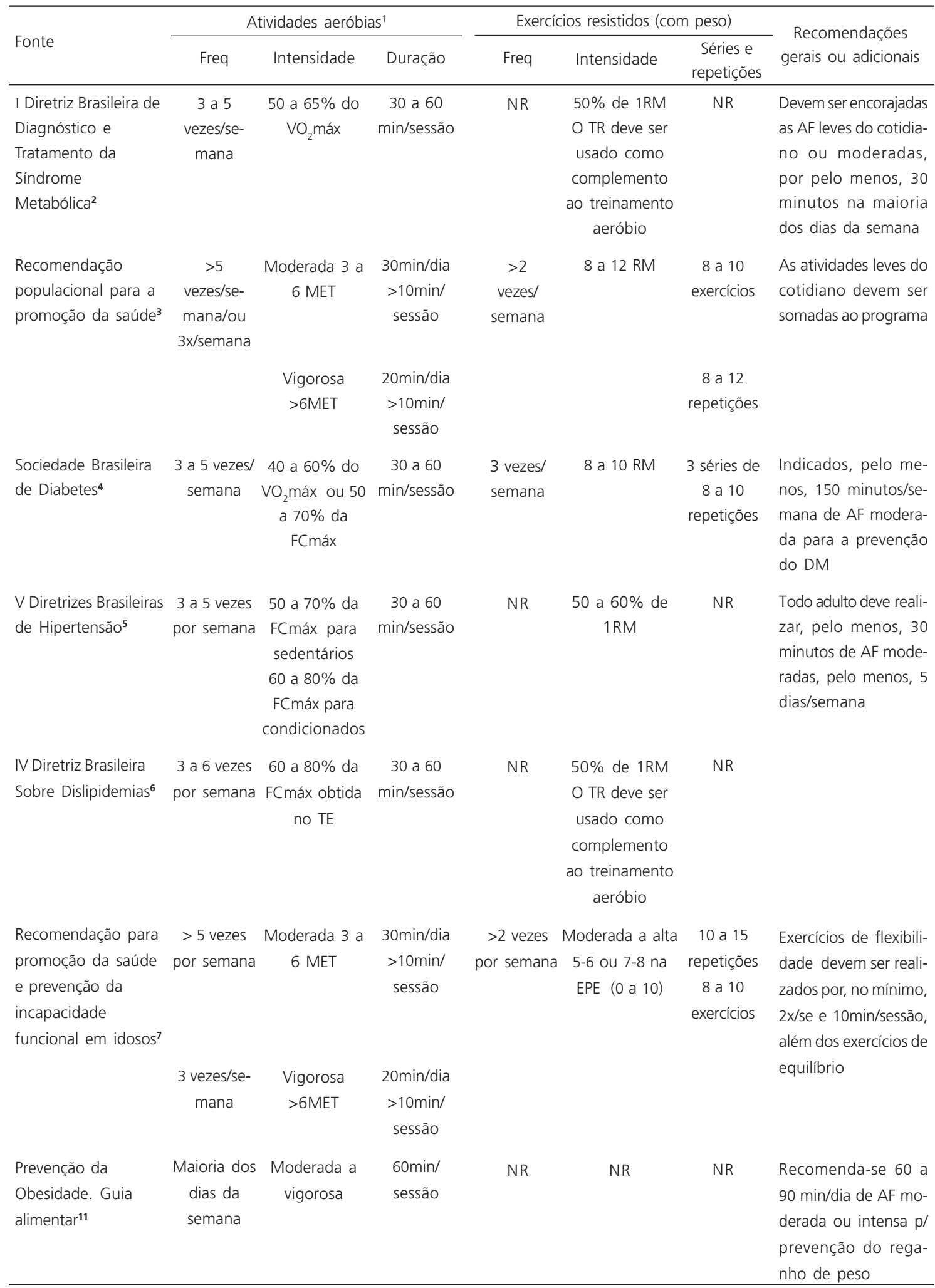

${ }^{1}$ caminhada, corrida, ciclismo, natação. Freq: freqüência; TE: teste ergométrico; FCmáx: freqüência cardíaca máxima obtida por teste ergométrico ou pela fórmula 220-idade; NR: não relatado; $\mathrm{VO}_{2}$ máx: consumo máximo de oxigênio; AF: atividade física; RM: repetição máxima; MET: equivalente metabólico; min/sessão: minutos por sessão; EPE: escala de percepção de esforço; TR: treinamento resistido. 
escritas orientais desde 3000 anos a.C. Na Grécia antiga, o reconhecimento de que quantidades apropriadas de atividade física eram necessárias para viver com saúde foi publicado nas chamadas leis da saúde, há cinco séculos antes de Cristo. Entretanto, foi a partir do século 20, com a publicação de estudos epidemiológicos, que a relação entre atividade física e doenças crônicas não-transmissíveis se tornou fortalecida.

No estudo pioneiro, publicado por Morris et al. ${ }^{12}$, comparou-se a prevalência de Doença Arterial Coronariana (DAC) entre carteiros e trabalhadores de escritório do serviço postal de Londres. Foi observado que as atividades ocupacionais com maior gasto energético estavam associadas à menor taxa de morte por Doenças Cardiovasculares (DCV).

Outro estudo epidemiológico importante, que revelou forte associação entre atividade física e doenças foi desenvolvido por Paffenbarger et al. ${ }^{13}$. Os autores determinaram, por questionários, o nível de atividade física de 16936 indivíduos de 35 a 74 anos, ex-alunos da Universidade de Harvard, e observaram, ao longo de 16 anos de estudo, declínio progressivo em eventos coronarianos tanto fatais como não fatais, à medida que aumentava o gasto energético diário (menos de $500 \mathrm{kcal} / \mathrm{semana}$ a $3500 \mathrm{kcal} / \mathrm{semana}$ ). Além disso, as taxas de morte foram $25 \%$ a $35 \%$ mais baixas nos indivíduos com gasto energético com atividade física maior ou igual a $2000 \mathrm{kcal} / \mathrm{sema-}$ na, mesmo na presença de outros fatores de risco, como hipertensão, obesidade e fumo. Foi observado também que gastos adicionais tinham pouco ou nenhum efeito na incidência de DCV.

Nas últimas três décadas, numerosos trabaIhos científicos ${ }^{14-17}$ têm demonstrado associação inversa entre alto nível de atividade física ou aptidão física e risco de doenças cardiovasculares e seus fatores de risco metabólicos.

No estudo longitudinal, realizado por Blair et al. ${ }^{14}$, no período de 1971 a 1989, com o objetivo de quantificar a relação entre condicionamento cardiorrespiratório e doença cardiovascular, os autores avaliaram por teste ergométrico, 25341 homens e 7080 mulheres de 20 a 80 anos, subdivididos em três grupos: baixo (L), moderado $(\mathrm{M})$ e alto $(\mathrm{H})$ condicionamento.

Os principais achados foram: a taxa de óbito foi consideravelmente menor nos indivíduos altamente condicionados em relação aos moderados e nestes em relação aos de baixo condicionamento. $\mathrm{O}$ baixo condicionamento cardiorrespiratório foi preditor de doença cardiovascular, enquanto que o alto e o moderado condicionamento promoveram uma proteção aos outros fatores de risco associados (colesterol, presença de doença, hipertensão e tabagismo), ou seja, os homens altamente condicionados, mesmo com tais fatores de risco, apresentaram menores taxas de óbito, quando comparados aos de baixo condicionamento, que não apresentavam nenhum outro fator de risco de morte.

Mediante esses estudos, pressupõe-se que o baixo gasto energético e o baixo condicionamento cardiorrespiratório $\left(\mathrm{VO}_{2}\right.$ máximo) podem ser considerados fatores de risco isolados no desenvolvimento das doenças cardiovasculares.

No entanto, é importante ressaltar que, além desses dois fatores, as mudanças desfavoráveis na composição corporal, por exemplo, o acúmulo adiposo e a redução da massa magra, também estão associados ao maior risco de morbidade e mortalidade precoce.

Além de ser fator de risco cardiovascular independente, a obesidade associa-se a uma série de outros fatores, como dislipidemia, hipertensão arterial e Diabetes mellitus, os quais são considerados componentes diagnósticos da síndrome metabólica ${ }^{3}$.

Não apenas a obesidade central, mas o excesso de peso generalizado está relacionado à maior mortalidade, embora o peso ideal para garantir longevidade ainda não esteja estabelecido, pois pode variar com a idade, raça ou sexo ${ }^{15}$.

Calle et al. ${ }^{15}$, em estudo epidemiológico prospectivo durante 14 anos envolvendo 588369 mulheres e 457785 homens adultos americanos com idade superior a 30 anos, avaliaram a relação 
entre Índice de Massa Corporal (IMC) e o risco de morte por todas as causas em quatro subgrupos, caracterizados de acordo com tabagismo, raça, histórico de doença e idade.

Os resultados mostraram que o ponto mais baixo da curva entre índice de massa corporal e mortalidade foi observado no IMC entre 23,5 e $24,9 \mathrm{~kg} / \mathrm{m}^{2}$ nos homens e 22 e $23,4 \mathrm{~kg} / \mathrm{m}^{2}$ nas mulheres. Aumento significativo no risco de morte por doença cardiovascular foi observado a partir de $25 \mathrm{~kg} / \mathrm{m}^{2}$ nas mulheres e $26,5 \mathrm{~kg} / \mathrm{m}^{2}$ nos homens. Em todas as faixas etárias houve aumento do risco de morte associado ao aumento do IMC.

Além do acúmulo de tecido adiposo, a redução da massa muscular (sarcopenia) e, conseqüentemente, da força muscular pode estar associada à síndrome metabólica e ao maior risco cardiovascular por diversos mecanismos, tais como: redução da captação muscular de glicose e de ácidos graxos livres, propiciando resistência à insulina e dislipidemia; redução da taxa metabólica de repouso e do nível de atividade física diária, com conseqüente redução do gasto energético total e maior propensão à obesidade; redução de capilares sangüíneos, número e densidade mitocondrial e de enzimas oxidativas, com conseqüente diminuição da capacidade cardiorrespiratória ${ }^{18}$.

Em estudo realizado ${ }^{17}$ com o objetivo de avaliar a associação independente e conjunta da força muscular e da aptidão cardiorrespiratória e a prevalência de síndrome metabólica, em 8570 homens saudáveis com idade entre 20 e 75 anos, foi observado que tanto a força quanto a aptidão cardiorrespiratória foram associadas inversamente e de forma independente à prevalência de SM. Os autores concluíram também que a força e, conseqüentemente, a massa muscular têm efeitos protetores contra a prevalência de SM, mesmo entre homens com diagnóstico de sobrepeso e obesidade.

Os resultados dos estudos citados mostraram clara associação entre sedentarismo, acúmulo adiposo, baixa aptidão cardiovascular e muscular e mortalidade precoce. No entanto, além da lon- gevidade, a preocupação com a qualidade dos anos vividos, ou seja, com a manutenção do estado de independência funcional, também deve ser encarada na perspectiva de Saúde Pública.

\section{Associação entre atividade física e prevenção da incapacidade funcional}

Nos Estados Unidos, calcula-se que entre os idosos, 9,1\% requerem assistência para a realização das Atividades da Vida Diária (AVD) básicas, 16,7\% necessitam de algum tipo de ajuda em atividades simples como fazer compras e cuidar da casa. Entre os indivíduos com idade acima de 75 anos, apenas 14,5\% são capazes de viver sozinhos, embora bastante limitados em realizar atividades como se inclinar, subir e descer degraus, ajoelhar-se, alcançar objetos, calçar sapatos, fazer atividades manuais, atravessar a sala caminhando e andar um quarteirão ${ }^{19}$.

Em pesquisa realizada na América Latina ${ }^{20}$, constatou-se que uma de cada quatro pessoas acima de 60 anos informou ter dificuldade no desempenho das AVD. No Brasil21 ${ }^{21}$ cerca de 1/4 dos indivíduos com idade superior a 50 anos referem ter limitação ou dificuldade para fazer as suas atividades habituais, por causa de algum problema de saúde ou incapacitação.

Estimativas americanas apontam que as pessoas idosas e funcionalmente dependentes contribuem com $46 \%$ dos gastos em saúde, porém correspondem a apenas $20 \%$ da população idosa no país. Além disso, os idosos funcionalmente dependentes gastam 5 mil dólares a mais por ano, comparados aos independentes ${ }^{22}$.

Os idosos constituem o grupo mais suscetível à incapacidade funcional. Dentre os fatores que explicam esse quadro destacam-se a maior prevalência de doenças e a redução do nível de atividade física, que ocorre nessa faixa etária6.

Assim, o sedentarismo e o aumento no número de doenças crônicas, freqüentemente, criam um círculo vicioso: doenças e inabilidade reduzem o nível de atividade física que, por sua vez, predis- 
põem o indivíduo ao maior risco de doenças e à incapacidade funcional.

Além disso, as mudanças fisiológicas, morfológicas e funcionais que ocorrem durante o processo natural de envelhecimento podem interferir negativamente na capacidade funcional desses indivíduos. As mais importantes relacionadas à independência funcional são as modificações na adiposidade corporal, na força muscular, na capacidade aeróbica e na flexibilidade ${ }^{11,22,23}$.

Estima-se que a partir dos 40 anos ocorra acúmulo de $1 \mathrm{~kg}$ de gordura, perda de $12 \%$ a $14 \%$ da força e cerca de $5 \%$ da massa muscular por década, com declínio mais rápido após os 65 anos e particularmente nos membros inferiores ${ }^{23}$.

A redução de massa muscular resulta em redução da força e da potência muscular, em perda de equilíbrio e, conseqüentemente, em maior incidência de quedas e fraturas ${ }^{24}$. Ainda, a realização de atividades simples, como levantar-se da cadeira, abrir torneiras, carregar compras, requer um nível mínimo de força muscular25.

Os mecanismos responsáveis pela perda de massa muscular no envelhecimento não estão totalmente esclarecidos; contudo, existe a redução da área da fibra muscular bem como no número de fibras musculares. A sarcopenia associa-se também à queda do conteúdo mineral ósseo, à redução da taxa metabólica de repouso, ao aumento da massa corporal gorda e à menor capacidade aeróbia ${ }^{23}$.

A sarcopenia contribui para a redução do $\mathrm{VO}_{2}$ máximo por diferentes mecanismos centrais, que incluem redução do volume sangüíneo e da fração de ejeção do coração e periféricos, como a redução da extração de oxigênio pelos músculos ativos, redução da densidade capilar e do conteúdo mitocondrial ${ }^{26}$.

A combinação dos efeitos da idade, da redução do nível de atividade física e do aumento da adiposidade faz com que o declínio do $\mathrm{VO}_{2}$ máximo seja de, aproximadamente, $10 \%$ a $14 \%$ por década, tanto para homens quanto para as mulheres ${ }^{27}$.
Estima-se que valores próximos a 15 e $16 \mathrm{~mL} / \mathrm{kg} / \mathrm{min}$ sejam necessários para a manutenção da independência e da capacidade funcional. Portanto, o nível adequado de capacidade aeróbia é essencial para que o indivíduo consiga caminhar, desenvolver atividades recreacionais e praticar esportes ${ }^{28}$.

A redução da flexibilidade e do equilíbrio também estão associadas a perdas das funções em várias AVD e podem ser uma das causas de dependência motora em indivíduo idoso. A flexibilidade do quadril, representada principalmente pela flexibilidade dos isquiotibiais e dos paravertebrais, declina $20 \%$ a $30 \%$ entre 20 e 70 anos, com uma queda mais acentuada aos 80 anos $^{29}$. O tempo de equilíbrio mantido por jovens é de cerca de 22 segundos contra apenas 13 segundos nos idosos ${ }^{30}$.

Observa-se, portanto, que a possibilidade de prevenir ou retardar a incapacidade funcional da população está diretamente associada à melhora da condição neuromotora e musculoesquelética (força, flexibilidade e equilíbrio) e cardiorrespiratória $\left(\mathrm{VO}_{2}\right.$ máximo), obtida pelo estilo de vida ativo.

\section{PERSPECTIVAS FUTURAS}

A limitação primária de muitos dados que ligam a atividade física à morbidade e à mortalidade por doenças crônicas é que poucos estudos randomizados com desenho adequado têm sido conduzidos. A maioria deles analisa apenas os efeitos do volume de atividade física (traduzido pelo gasto energético total) sobre vários desfechos clínicos. Portanto, mais estudos devem ser conduzidos para mostrar, de forma independente, a contribuição da intensidade, da duração ou da freqüência dos exercícios sobre tais desfechos.

Além disso, mesmo com o crescimento do número de informações, publicações científicas e consensos em atividade física, o sedentarismo entre os indivíduos adultos e idosos permanece alto e demonstra que a adoção do estilo de vida ativo, embora benéfica, pode ser difícil de ser 
realizada. As pesquisas devem se concentrar em conhecer melhor as barreiras e os fatores que facilitam a adesão e manutenção dos indivíduos aos programas de exercício.

Ainda, a prática de atividade física é somente um dos diversos comportamentos que afetam a saúde. Muitos estudos epidemiológicos com atividade física integram os aspectos dietéticos e psicológicos nos programas os quais podem interferir nos resultados obtidos.

\section{CONSIDERAÇÕ ES FINAIS}

A prática de atividade física pode prevenir o surgimento precoce, atuar no tratamento de diversas doenças metabólicas e interferir positivamente na capacidade funcional de adultos e idosos.

Os mecanismos que ligam a atividade física à prevenção e ao tratamento de doenças e à incapacidade funcional envolvem principalmente a redução da adiposidade corporal, a queda da pressão arterial, a melhora do perfil lipídico e da sensibilidade à insulina, o aumento do gasto energético, da massa e da força muscular, da capacidade cardiorrespiratória, da flexibilidade e do equilíbrio.

No entanto, existe importante distinção entre atividade física para prevenção de doenças crônicas, para o bom condicionamento físico e para o tratamento de doenças, associada tanto ao tipo quanto à freqüência, à intensidade e à duração das atividades realizadas.

\section{COLABORADORES}

C.F COELHO participou da busca dos artigos nas bases de dados e da redação do artigo. R.C. BURINI participou da redação e da revisão do artigo pronto.

\section{REFERÊ N CIAS}

1. Barreto SM, Pinheiro $A R O$, Sichieri R, Monteiro CA, Batista Filho M, Schimidt Ml, et al. Análise da estratégia global para alimentação, atividade física e saúde, da Organização Mundial da Saúde. Epidemiol Serv Saúde. 2005; 14(1):41-68.

2. Brasil. Ministério da Saúde. Vigilância de Doenças e Agravos não Transmissíveis (DAnT). Brasília. [acesso 2007 jan 1]. Disponível em: <http://portal. saude.gov.br/portal/svs/area.cfm?id_area=448>.

3. I Diretriz Brasileira de Diagnóstico e Tratamento da Síndrome Metabólica. Arq Bras Cardiol. 2005; 84 (Suppl I):3-28. doi: 10.1590/S0066-782X200 5000700001

4. U.S. Department of Health \& Human Service. Physical activity and health: a report of surgeon general [cited 2007 Oct 9]. Available from: <http:/ /www.surgeongeneral.gov/library/reports.htm>.

5. Pate RR, Pratt M, Blair SN, Haskell WL, Macera CA, Bouchard C, et al. Physical Activity and Public Health. A recommendation of the Centers for Disease Control and Prevention and the American College of Sports Medicine. JAMA. 1995; 273(5): 402-7.

6. Haskell WL, Lee I, Pate LL, Powell KE, Blair SN, Franklin BA, et al. Physical Activity and Public Health. Updated Recommendation for Adults From the American College of Sports Medicine and the American Heart Association. Circulation. 2007; 116(9):1081-93.

7. U.S. Department of Health and Human Services. Dietary guidelines for americans, [cited 2007 Oct 1]. Available from: <http://www.healthierus.gov/ dietaryguidelines $>$.

8. Tratamento e acompanhamento do Diabetes mellitus. Diretrizes SBD. 2006. Disponível em: $<$ http://www.diabetes.org.br/politicas/abdonline. php>.

9. V Diretrizes Brasileiras de Hipertensão. Sociedade Brasileira de Hipertensão. [acesso 2006 jan 12]. Disponível em: <http://www.sbh.org.br/documentos/>.

10 IV Diretriz brasileira sobre dislipidemias e prevenção da aterosclerose. Departamento de Aterosclerose da Sociedade Brasileira de Cardiologia. Arq Bras Cardiol. 2007; 88(Suppl I):2-19. doi: 10.1590/S006 6-782X2007000700002

11. Nelson ME, Rejeski JW, Blair SN, Duncan PW, Judge JO, King AC, et al. Physical Activity and Public Health in Older Adults: Recommendation from the American College of Sports Medicine and the American Heart Association. Med Sci Sports Exerc. 2007; 39(8):1435-45.

12. Morris JN, Heady JA, Raffle PAB, Roberts CG, Parks JW. Coronary heart disease and physical activity of work. Lancet.1953; 2:1053-7.

13. Paffenbarger RS, Ryde, RT, Wing AL, Hsieh C. Physical activity, all-cause mortality and longevity 
946 | C.F. COELHO \& R.C. BURINI

of college alumni. N Engl J Med.1986; 34(10): 605-13.

14. Blair SN, Kampert JB, Kohl HW, Barlow CE, Macera CA, Paffenbarger RS, et al. Influences of cardiorespiratory fitness and other precursors on cardiovascular disease and all-cause mortality in men and women. JAMA. 1996; 276(3):205-10.

15. Calle EE, Thun MJ, Petrelli JM, Rodriguez C, Heath CW Jr. Body-mass index and mortality in a prospective cohort of U.S. adults. N Engl J Med. 1999; 341(15):1097-105.

16. Lee DC, Blair SN. Cardiorespiratoy fitness and stroke mortality in men. Med Sci Sports Exerc. 2002; 34(4):592-5.

17. Jurca R, Michael JL, Timothy SC, Conrade PE, Shannon JF, Carolyn EB, et al. Associations of muscle strength and aerobic fitness with Metabolic Syndrome in men. Med Sci Sports Exerc. 2004; 36(8):1301-7.

18. Ciolac EG, Guimarães GV. Exercício físico e síndrome metabólica. Rev Bras Med Esporte. 2004; 10(4): 319-24

19. Holland JG, Tanaka K, Shigematsu R, Nakagaichii M. Flexibility and physical functions of older adults: a review. JAPA. 2002; 10:169-206.

20. Organização Pan-Americana da Saúde. Anais da XXVI Conferência Sanitária Pan-Americana. 23-27 de set 2002; Washington (DC). [acesso 2007 out 1]. Disponível em: <http//www.who.int/dietphysicalactivity>.

21. Brasil. Ministério da Saúde. Inquérito domiciliar sobre comportamentos de risco e morbidade referida de doenças e agravos não transmissíveis: Brasil, 15 capitais e Distrito Federal. Brasília; 2004 [acesso 2007 jan 23]. Disponível em: <http://www.inca. gov.br/inquerito/docs/completa.pdf>.

22. Manini TM, Pahor M. Physical activity and maintaining physical function in older adults. BJSM. 2009; 43:28-3.
23. Silva TA, Frisoli AJ, Pinheiro MM, Szejnfeld VL. Sarcopenia associada ao envelhecimento: aspectos etiológicos e opções terapêuticas. Rev Bras Reumatol. 2006; 46(6):391-7. doi: 10.1590/S048 2-50042006000600006.

24. Pereira SRM, Buksman S, Perracini M, Py L, Barreto $\mathrm{KML}$, Leite VMM, et al. Quedas em idosos. Soc Bras Ger Gerontol [periódico na internet]. 2001; 2-9. Disponível em: <http://www.connectmed. com.br/amb/novo/projeto_diretrizes/quedas_ idosos.pdf $>$.

25. Kell TR., Bell G, Quinney A. Musculoskeletal fitness, health outcomes and quality of life. Sports Med. 2001. 31(12):863-73.

26. McGuire DK, Levine DB, Williamson JW, Snell PG, Blomqvist CG, Saltin B, et al. A 30-year follow-up of the Dalas best and training study. Circulation. 2000; 18:1350-6.

27. Ravagnani FCP, Coelho CF, Burini RC. Declínio do consumo máximo de oxigênio em função da idade de indivíduos do sexo masculino submetidos a teste ergoespirométrico. Rev Bras Cienc Mov. 2005; 13(3):23-8

28. Shephard RJ. Exercise and aging: extending independence for older adults. Geriatrics.1993; 48:61-4.

29. Ueno LM, Okuma SS, Miranda ML, Jacob Filho W, Lee Ho L. Análise dos efeitos quantitativos e qualitativos de um programa de educação física sobre a flexibilidade do quadril em indivíduos com mais de 60 anos. Motriz. 2000; 6(1):9-16.

30. Daley MJ, Spinks WL. Exercise, mobility and aging. Sports Med. 2000; 29(1):1-12.

Recebido em: 10/12/2007

Versão final reapresentada em: 2/8/2009 Aprovado em: 6/10/2009 